\title{
Web Services Based Information Retrieval Agent System for Cloud Computing
}

\author{
Yu Mon Zaw \\ University of Technology, \\ Yatanarpon Cyber City, \\ Mandalay, Myanmar
}

\author{
Nay Min Tun \\ Computer University, \\ Kyaing Ton, \\ Shan State,Myanmar
}

\begin{abstract}
Cloud computing is Internet based system development in which large scalable computing resources are provided "as a service" over the Internet to users and has attracted more and more attention from industry and research community. However, the concept of cloud computing does not provide facilities for the knowledge discovery and information retrieval; i.e. clouds need to be intelligent and autonomous. On the other hand, Web Service plays important role in Service Oriented Computing (SOC) in cloud environment. Retrieving desired specific information from Web Services on cloud environment cannot be done by single Web Service. So, there should be a possibility to combine existing services together in order to fulfil the request. To compose Web Services, Agents can give great help. Therefore, this research mainly focus on providing a framework for retrieving information from Cloud using composite Web Services by means of Multi-Agent System.
\end{abstract}

Keywords: Cloud Computing; Multi-Agent; Web Services; Service Oriented Computing; Information Retrieval

\section{INTRODUCTION}

Cloud computing provide elastic services, high performance and scalable data storage to a large and everyday increasing number of users. Cloud computing enlarged the arena of distributed computing systems by providing advanced Internet services that complement and complete functionalities of distributed computing provided by the Web, Grid computing and peer-to-peer networks. In fact, Cloud computing systems provide large-scale infrastructures for high performance computing that are dynamically adapt to user and application needs. [1]

According to the current situation, most of ongoing works or researches are intended at developing the techniques and constructing cloud platforms, such as Amazon, Google AppEngine, Microsoft Azure, and manjrasoft Aneka. [2] We need more researches in information retrieval and knowledge discovery area. As Web Services play the major role in Cloud Environment since Cloud's main feature is also "as-aservice", to achieve the user desired information correctly and completely may depend on numerous Web Services' supports.

Web Services are considered as self-contained, self describing, modular applications that can be published, located, and invoked across the Web. Amount of products and services available now on the Web increases dramatically and goes beyond user's ability to analyze them efficiently. At the same time the number of potential customers available via the Internet also increases significantly and starts to be beyond service providers' ability to perform efficient targeted marketing. In particular, if no single Web service can satisfy the functionality required by a user, there should be a possibility to combine existing services together in order to fulfill the request. [3]

At the same time, multi-agent systems (MAS) represent another distributed computing paradigm based on multiple interacting agents that are capable of intelligent behavior. Multi-agent systems are often used to solve problems by using a decentralized approach where several agents contribute to the solution by cooperating one each other. One key feature of software agents is the intelligence that can be embodied into them according to some collective artificial intelligence approach that needs cooperation among several agents that can run on a parallel or distributed computer to achieve the needed high performance for solving large complex problems keeping execution time low. [1]

Therefore, for the Cloud-wide Information Retrieval system based on Web Services, it is sure that not a single Web Service can fulfill the user needs. To get the complete and desired information results, numerous related Web Services should be cooperated. In this case, we propose to get the help of multi- agents systems.

The remainder of this paper is organized as follows. In the next section, we will introduce the background knowledge and theory of Cloud Computing, Service Oriented Computing (SOC), Web Services and Agent Computing. Section 3 describes the related work. Section 4 discusses about our proposed framework. We introduce our system components, their functions and natures in Section 5. Section 6 presents proposed Algorithms for our Medical IR MultiAgent System. The paper concludes in Section VII with titled Conclusion.

\section{BACKGROUND \\ 2.1 Cloud Computing}

Cloud computing [9] is a model for enabling convenient, on-demand network access to a shared pool of configurable computing resources (eg. Networks, servers, storage, applications, and services) that can be rapidly provisioned and released with minimal management effort or service provider interaction. (by U.S. NIST (National Institute of Standards and Technology))

Five essential elements of cloud computing are:

- On-demand self-service

- Broad network access

- Resource pooling

- Rapid elasticity

- Measured Service 

are:

- Software as a Service (SaaS)- Cloud consumers release their applications on a hosting environment, which can be accessed through networks from various clients (e.g. web browser, PDA, etc.) by application users. Examples of SaaS include SalesForce.com, Google Mail, Google Docs, and so forth.

- Platform as a Service (PaaS)- PaaS is a development platform supporting the full software Lifecycle which allows cloud consumers to develop cloud services and applications(e.g. SaaS) directly on the PaaS cloud. Hence the difference between SaaS and PaaS is that SaaS only hosts completed cloud applications whereas PaaS offers a development platform that hosts both completed and in-progress cloud applications. Eg. Google App Engine.

- Infrastructure as a Service (IaaS)-Cloud consumers directly use IT infrastructures (processing, storage, networks, and other fundamental computing resources) provided in the IaaS cloud. Virtualization is extensively used in IaaS cloud in order to integrate/decompose physical resources in an ad-hoc manner to meet growing or shrinking resource demand from cloud consumers.

Four cloud deployment models have been defined in the Cloud community:

- Private cloud.-The cloud infrastructure is operated solely within a single organization, and managed by the organization or a third party regardless whether it is located premise or off premise. Academics often build private cloud for research and teaching purposes.

- Community cloud-Several organizations jointly construct and share the same cloud infrastructure as well as policies, requirements, values, and concerns. The cloud infrastructure could be hosted by a third-party vendor or within one of the organizations in the community

- Public cloud-The public cloud is used by the general public cloud consumers and the cloud service provider has the full ownership of the public cloud with its own policy, value, and profit, costing, and charging model.

- Hybrid cloud.-The cloud infrastructure is a combination of two or more clouds (private, community, or public) that remain unique entities but are bound together by standardized or proprietary technology that enables data and application portability.

\subsection{Cloud and Service Oriented Computing (SOC)}

The encapsulation, componentization, decentralization, and integration capability provided by SOC are substantial: they provide both architectural principles and software specifications to connect computers and devices using standardized protocols across the Internet.
In fact, the notion of Cloud is more or less based on the evolving development on SOC, in particular the SaaS service model.

Advances in SOC can benefit Cloud Computing in several ways:

- Service Description for Cloud Services- Web Service Description Language (WSDL) and the REST protocol are two widely used interface languages to describe Web Services. They have been utilized to describe Cloud API specification.

- $\quad$ Service Discovery for Cloud Services- Various service discovery models can be leveraged for cloud resource discovery, selection and service-level agreement verification.

- Service Composition for Cloud Services- Since Web Services are born to compose business applications, a great deal of research in this area can be leveraged for cloud services integration, collaboration, composition.

- Service Management for Cloud ServiceResearch and practices in SOA governance and services management can be adapted and reused in the cloud infrastructure management.

\subsection{Web Services}

Web Service[4] is an accessible application that other applications and humans as well, can automatically discover and invoke. An application is a Web Service if it is

1) independent as much as possible from specific platforms and computing paradigms;

2) developed mainly for inter organizational situations rather than for intra-organizational situations; and

3) easily composable (i.e., its composition with other Web Services does not require the development of complex adapters).Web Services are, in practice, transient and stateless processes that exist only during service execution, which is triggered by a request coming from a consumer, or client. Services are instantiated to perform specific tasks, thus facilitating scalable, concurrent service provision. The design of a Web Service is usually defined as a clearly articulated workflow, for the sake of reliability and quality of service.

Though Web Services has many advantages, but still there are certain problems which need to be addressed. These are:

1) Provided resources and services are not in machine understandable form, these are in human understandable form.

2) The representation of resources and services on the web are unstructured and they are loosely related to each other.

3) Searching resources and services on the web at present is keyword based; no semantics of the resources are used. So by using some popular keywords, web page owner can make his page mostly retrieval with irrelevant results and

4) Interoperability between toolkits. 


\subsection{Agent Computing}

An agent [1] is a computational entity that acts on behalf of another entity (or entities) to perform a task or achieve a given goal. Agent systems are self-contained software programs embodying domain knowledge and having ability to behave with a specific degree of independence to carry out actions needed to achieve specified goals. They are designed to operate in a dynamically changing environment.

Agents typically include a set of features. The main features of agents include the following:

- Autonomy: the capacity to act autonomously to some degree on behalf of users or other programs also by modifying the way in which they achieve their objectives.

- Pro-activity: the capacity to pursue their own individual set goals, including by making decisions as result of internal decisions.

- Re-activity: the capacity to react to external events and stimuli and consequently adapt their behavior and make decisions to carry out their tasks.

- Communication and Cooperation: the capacity to interact and communicate with other agents (in multiple agent systems), to exchange information, receive instructions and give responses and cooperate to fulfill their own goals.

- Negotiation: the capability to carry out organized conversations to achieve a degree of cooperation with other agents.

- Learning: the ability to improve performance and decision making over time when interacting with the external environment.

\section{RELATED WORK}

Yue-San Chang, Chao-Tung Yang and Yu-Cheng Luo presented an Ontology based Agent Generation for Information Retrieval on Cloud Environment. [2] While user submitting a flat-text based request for retrieving information on a based on predefined ontology and reasoning rule, and then be translated to a Mobile Information Retrieving Agent Description File (MIRADF) that is formatted in a proposed Mobile Agent Description Language (MADF). A generating agent, named MIRA-GA, is also implemented to generate a MIRA in accordance with MIRADF.

G.Vadivelou, E.Ilavarasan and M.S.Yasmeen presented an agent and ontology based approach that supports the semi-automatic composition of Web Services.[3] This paper provided the way to select an optimal composition of services and it also propose a framework for Semi-Automatic Web Services Composition.

Energy-Saving Information Multi-agent System with Web Services for Cloud Computing was given by ShengYuan Yang, Dong-Liang Lee, Kune-Yao Chen and ChunLiang Hsu from St. John's University in Taiwan. [5] It employs the concept of SQL IC to construct the operational interface of cloud database as a data warehouse. It presented the three-stage intelligent decision processing strategy with four agents: Interface agent, Data Mining agent, Reasoning agent and Web-Service-Based Information Agent System (WIAS).
Vishal Jain proposed the information retrieval practical model through the multi-agent system with data mining in a cloud computing environment. [6] $\mathrm{He}$ recommended that users should ensure that the request made to the IaaS is within the scope of integrated data warehouse and is clear and simple. In that research model/ architecture, the use of cloud computing allows the users to retrieve meaningful information from virtually integrated data warehouse that reduces the costs of infrastructure and storage.

\section{THE PROPOSED FRAMEWORK}

We propose a framework for Web Services Based Information Retrieval Agent System for Cloud Computing Environment. The proposed system framework is intended to apply in Medical field. We have already assumed that a private Medical Cloud Environment was been founded. In that environment, a number of hospitals, clinics and health care services are hosted and are providing Web Services. Each Web Service of a specific hospital offers specialists (doctors) information worked at that hospital by numerous Web Methods. By using our Medical IR multi-agent System, users (patients) can easily search the desired information by day (Monday, Tuesday,...), by time $(1 \mathrm{pm}-4 \mathrm{pm}, \ldots)$, by doctor's name (Prof: Dr. Nay Win,...), by specific clinic (Asia Royal, $\mathrm{SSC}, .$.$) and by disease type (Liver, Lung, \mathrm{OG}, . .$. ).

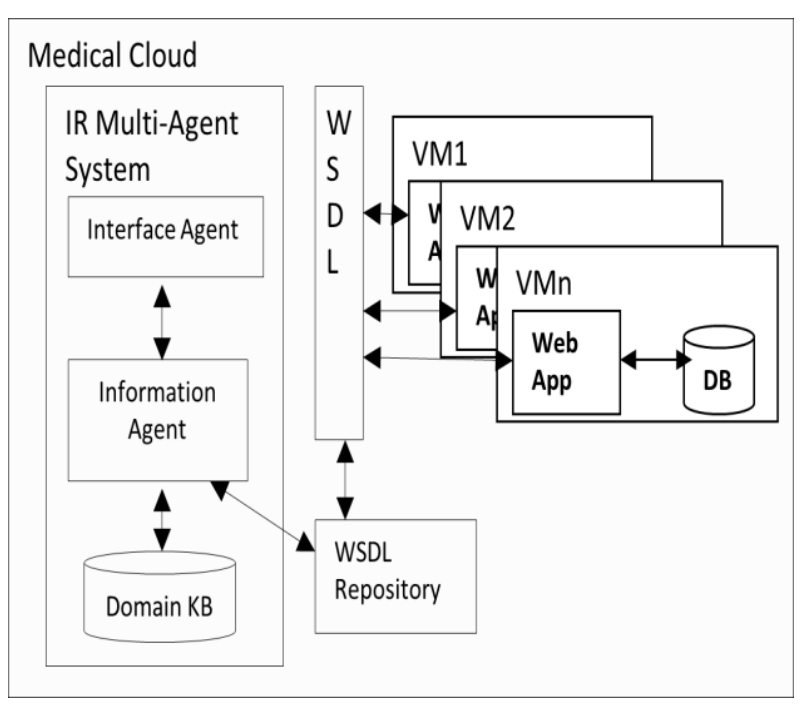

Figure 1. Medical IR Multi-Agent System Architecture

\section{SYSTEM COMPONENTS AND THEIR FUNCTIONS}

- The system will start from Interface Agent.

- $\quad$ Receiving queries from end users to search required information from Cloud databases and showing back the queries result may be done by interface agent in this proposed system.

- The duty of Interface Agent in this system is to receive queries from end users, prepare the queries into a format match for Information Agent's working style, pass the well formatted data to Information Agent and to show back the queries results. 
- Information Agent will play as a major role and it uses specific Domain Knowledge Base for cloudwide service composition and then decides which services can be a perfect match for the user queries.

- Domain Knowledge Base is a repository which stores system associated rules and conditions.

- WSDL Repository stores Web Service Description files published by various web applications hosted in the Cloud.

- Several web application systems from VMs (Virtual Machines) support services for Information Retrieval purpose.

- One VM represents one hospital.

- Each VM contains a web application (web site) for each hospital which desires to coordinate with our cloud-wide Medical IR Multi-agent System.

\section{PROPOSED ALGORITHMS FOR MEDICAL IR MULTI-AGENT SYSTEM}

- User Request is defined as Req.

- $\quad$ Refined Request is defined as RefineReq.

- The Returned Result from each Web Service is defined as ResWS

- $\quad$ ResWS1, ResWS2,..., ResWSn $€$ ResWS

- Extracted Rules associated with user requests is defined as R.

- WS is the set of Web Services published in WSDL Repository.

- WS1, WS2, ...,WSn $\epsilon$ WS

- $\quad$ Selection Web Services is defined as SelectedWS.

- $\mathrm{AG}$ is the set of all agents in the System.

- Interface $A G$, InfoAG $€ A G$

- Interface Agent is defined as InterfaceAG.

- Information Agent is defined as InfoAG.

Begin

FinResult $\leftarrow$ NULL.

User sends Req to InterfaceAG.

RefineReq $\leftarrow$ Req refined by Interface AG.

InterfaceAG sends RefineReq to InfoAG.

InfoAG extracts $\mathrm{R}$ appropriate with RefineReq.

InfoAG calls SelectWSAlgo(R).

WSŁSelectedWS.

While not receiving the FinResult from InfoAG

InfoAG sends RefineReq to WS1, WS2, .., WSn

$\epsilon \mathrm{WS}$.
For All WS1,WS2,..,WSi,..,WSn

If WSi can solve RefineReq

It will return ResWSi to

InfoAG.

Else

Return NULL.

End If

FinResult $\leftarrow$ FinResult + ResWSi.

End For

End While

InfoAG sends back FinResult to InterfaceAG.

Interface AG shows the FinResult to User.

End

SelectWSAlgo(R)

Begin

SelectedWS $\leftarrow$ NULL

ForAll ItemR in R

For All WS1,WS2,..,WSi,..,WSn in WS

If WSi content matches ItemR

SelectedWS $\leftarrow$ SelectedWS + WSi

Else

SelectedWS $\leftarrow$ SelectedWS + NULL

End If

End For

End For

Return SelectedWS.

End

\section{CONCLUSION}

A Web Services Based Information Retrieval Agent System for Cloud Computing is proposed. Efficiently composed cloud Web Services using multi-agents features can give new form for cloud wide information retrieval systems. The proposed system will become an intelligent way for searching or retrieving information from Cloud environment. By implementing the propose system, it can give a good hand for the public to get the desired specialists' schedule completely and perfectly at one sitting and can make the right choice with their current situations. Moreover, this framework can be applied in other domain area efficiently. 


\section{REFERENCES}

[1] Talia, D. 2012. Cloud Computing and Software Agents : Towards Cloud Intelligent Services.

[2] Chang, Y. S., Yang, C. T., and Luo, Y. C. 2011. An ontology based agent generation for information retrieval on cloud environment, Journal of Universal Computer Science, vol. 17, no. 8, 2011.

[3] Vadivelou, G., Ilavarasan, E., and Yasmeen, M. S. 2011, QoS based semi-automatic web service composition using multi-agents systems, IJCSET, Vol 1, Issue 7, pp. 381-386, August 2011.

[4] Curbera, F., Khalaf, R., Mukhi, N., Tai, S., and Weerawarana, S. 2003, The Next Step in Web Services, Comm. ACM, vol. 46, no. 10, October 2003.

[5] Yang, S. Y., Lee, D. L., Chen, K. Y., and Hsu, C. L 2011., Energy-saving information multi-agent system with web services for cloud computing, SUComS 2011 , CCIS 223, pp. 222-233.

[6] Jain, V. 2012, Information retrieval through multi-agent system with data mining in cloud computing, J.Comp.Tech.Appl, Vol 3 (1), pp. 62-66, IJCTA.

[7] Wang, S., Zheng, Z., Sun, Q., Zou, H., and Yang, F. 2011, Cloud model for service selection, IEEE INFOCOM Workshop on Cloud Computing.

[8] Pejman, E., Rastegari, Y., .Majlesi Esfahani , P., and Salajegheh, A. 2012, Web Service Composition Methods: A Survey, IMECS 2012 Vol I.

[9] Dillon, T., Wu, C., and Chang, E. 2010, Cloud Computing Issues and Challenges, 24th IEEE International Conference on Advanced Information Networking and Applications. 